\title{
CROWNED DENS SYNDROME
}

\author{
Munasinghe CS, De Silva A \\ Department of Radiology, National Hospital of Sri Lanka
}

DOI: http://doi.org/10.4038/sljr.v3i1.38

Keywords: Crowned dense syndrome, Calcium pyrophosphate dehydrate (CPPD)

\section{Introduction}

Crowned dens syndrome is a rare disease entity, which radiologically shows calcification at cruciform ligament around the odontoid process and clinically presents as acute cervico-occipital pain, fever and neck stiffness accompanied by a biological inflammatory reaction. This condition usually requires medical management only, however if an inflammatory pannus causes cervical cord compression it may require surgical decompression.

\section{Case report}

An 84 year old male presented to the casualty unit with recent onset mild fever, occipital headache and posterior neck pain. There was no history of trauma but physical examination revealed neck stiffness. Non contrast CT scan of the head was unremarkable apart from chronic periventricular microangiopathic changes and cerebral involution. However, on upper cervical spine, there was osteophytic lipping at the tip of the dens (Fig.1) and superior to the anterior arch of the atlas.

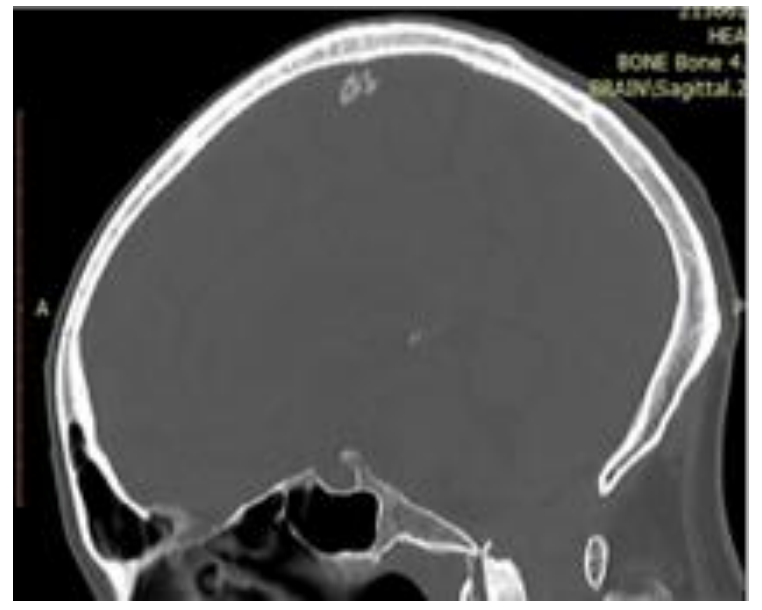

Figure 1: Sagittal CT - osteophytic lipping at the tip of the dens

No bone erosion was identified. There was presumed soft tissue pannus posterior to the dens and the craniocervical junction (Fig.2) which caused mild cord flattening although a small amount of CSF was still present mainly lateral to the cord. Coronal CT images showed atlantoaxial synovial calcifications in a crown like distribution around the odontoid process (Fig.3).

Corresponding Author: Munasinghe CS <chathu782000@yahoo.co.in>

https://orcid.org/0000-0002-8614-558X

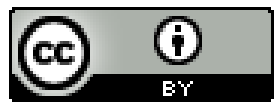

This is an open-access article distributed under the terms of the Creative Commons Attribution 4.0 International License, which permits unrestricted use, distribution and reproduction in any medium provided the original author and source are credited. 


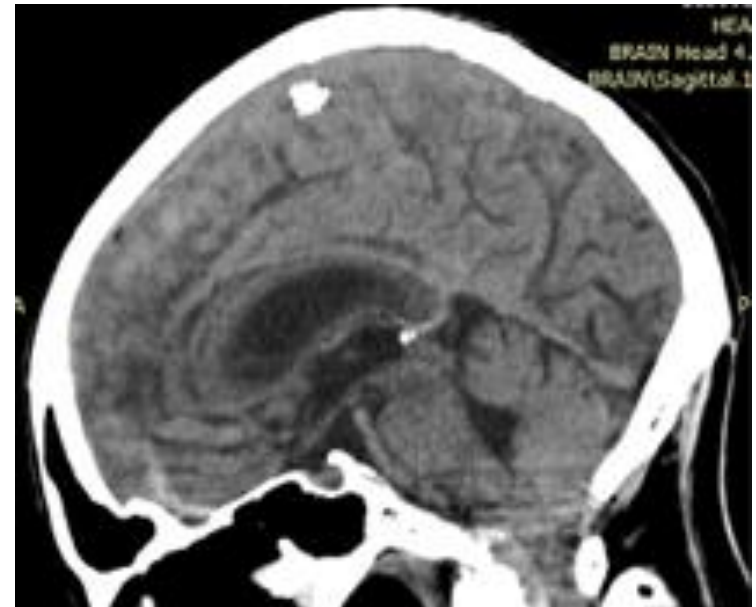

Figure 2: Sagittal CT (non contrast) hyperdense soft tissue pannus posterior to the dens

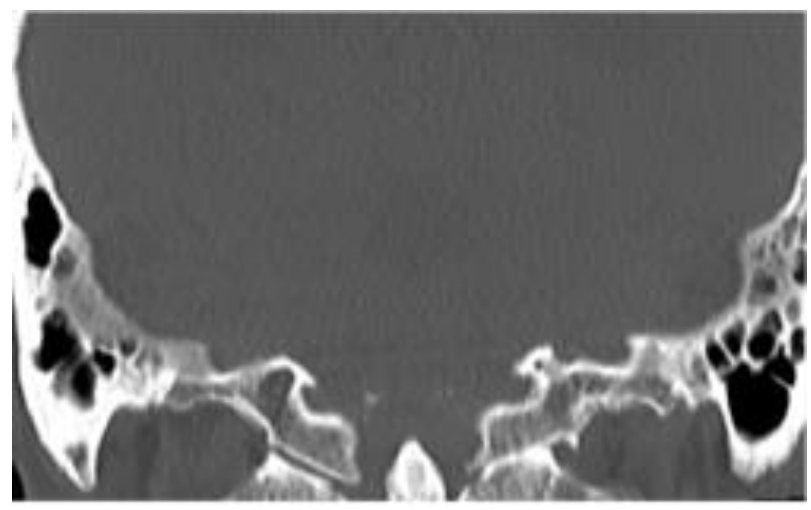

Figure 3: Coronal CT - atlantoaxial synovial calcifications in a crown like distribution around the odontoid process

MRI scan of the cervical spine (pre and post IV gadolinium enhanced) was done 2 days after the CT scan. By this time patient had developed marked restriction of neck motion and drowsiness. On physical examination, passive cervical spine movements were significantly reduced without focal neurologic deficit. Patient's vital signs were stable. There was elevated C-reactive protein (CRP) of $4 \mathrm{mg} / \mathrm{L}$ while other blood tests were unremarkable.
On MRI, there was enhancing soft tissue material surrounding the tip of the odontoid process. Posteriorly this measured up to $5.6 \mathrm{~mm}$ in thickness, resulting in mild compression of the cervico-medullary junction that demonstrated a faintly undulated anterior surface (Fig. 4). No significant flattening was seen. However, there was conspicuous osseous oedema involving the odontoid process and upper body of $\mathrm{C} 2$ and to a lesser extent right lateral masses of $\mathrm{C} 1$, with involvement of the anterior arch of $\mathrm{C} 1$ as well (Fig. 5). Faint osseous erosions were seen at the $\mathrm{C} 1 / 2$ articulation as a result. The cervical and upper thoracic cord demonstrated normal signal throughout. There was faint oedema involving the paraspinous muscles in the upper half of the cervical spine. There were no visible rim enhancing, epidural or paraspinous collections. Findings were considered likely to reflect active calcium pyrophosphate dehydrate (CPPD) deposition indicating crowned dens disease although an infective aetiology (given the history of fever) was also considered a possibility.

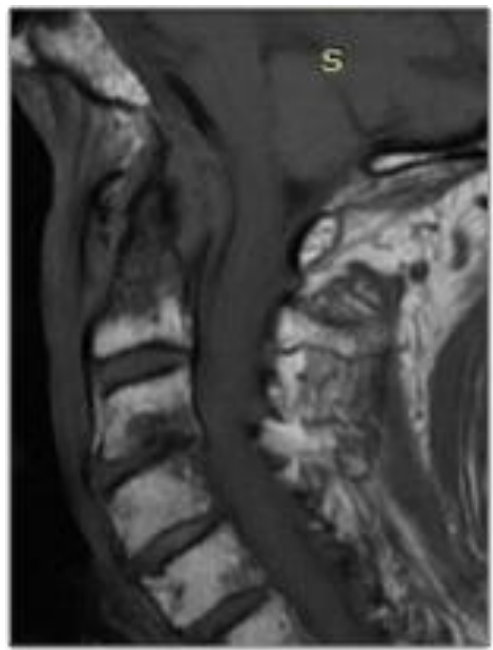

Figure 4: MRI Sagittal T1W post IV gadolinium - enhancing soft tissue material at the tip of the dens with compression of the cervico-medullary junction 
Patient was treated with a short course of combination of NSAID and prednisolone and the clinical symptoms markedly improved.

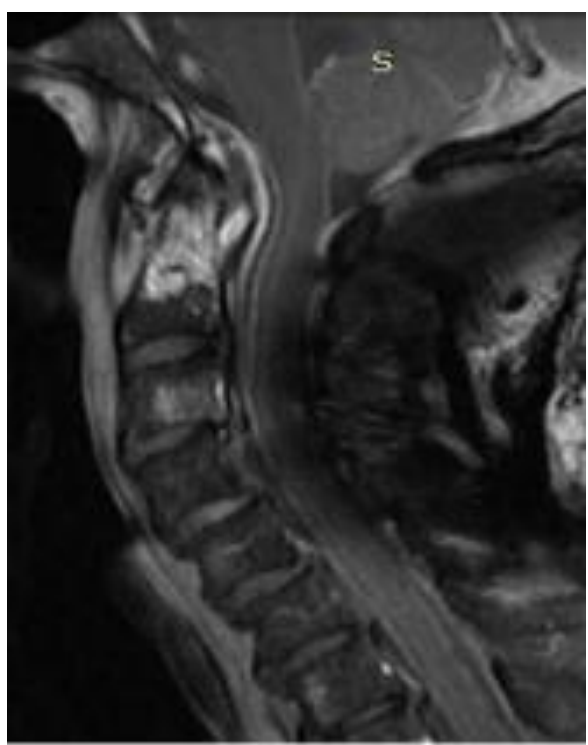

Figure 5: MRI sagittal STIR - osseous edema of the odontoid process, upper body of $\mathrm{C} 2$ and the anterior arch of $\mathrm{C} 1 \mathrm{junction}$

\section{Discussion}

It is believed that crowned dens syndrome is commoner than previously recognized, especially in elderly patients. The clinical manifestations of this case include severe posterior neck pain of acute onset, significant restriction of neck rotation and elevation of serum CRP that could be clinically mistaken as spondyloarthritis around atlantoaxial joint. The duration of pain in crowned dense syndrome varies from few days to several weeks, the location can be from suboccipital area to inferior posterior neck, and the characteristics of pain are also diverse from mild neck discomfort to sleep-breaking severe pain. ${ }^{1}$
Most of the crystalline deposits are CPPD crystals or hydroxyapatite crystal. There are no specific symptoms when the crystal size is small, but as it grows, it causes chronic cervical pain or spinal cord compression. Calcification can occur at not only cruciform ligament but also transverse, alar and apical ligaments. Especially when CPPD crystal deposition occurs at ligamentum flavum, compressive cervical myelopathy can be presented.2 Dirheimer et al. performed plain radiographs in 27 patients who had articular chondrocalcinosis but were neurologically asymptomatic, and demonstrated that syndesmo-odontoid joint calcification was found in 12 patients (44\%). ${ }^{3}$ Constantin et al. performed CT scan in 21 patients with articular chodrocalcinosis, and found that 14 patients had calcifications at transverse ligament and 5 patients out of the 14 presented with neck pain.

\section{Conclusion}

Crowned dens syndrome is diagnosed in the presence of acute and severe neck pain with marked restriction of neck motion, particularly in rotation, elevated inflammatory indicators and calcium deposition around the odontoid process detected by $\mathrm{CT}$, in the absence of history of trauma and with exclusion of other inflammatory diseases and tumors. It is important for the clinicians to be aware of clinical feature of CDS in order to avoid unnecessary invasive management strategies such as lumbar puncture or biopsy. 


\section{References}

1. Constantin A, Bouteiller G. Acute neck pain and fever as the first manifestation of chondrocalcinosis with calcification of the transverse ligament of the atlas. Five casereports with a literature review. Rev RhumEngl Ed. 1998;65:583-585.

2. Fye KH, Weinstein PR, Donald F. Compressive cervical myelopathy due to calcium pyrophosphate dihydratedeposition disease: report of a case and review of the literature. Arch Intern Med. 1999;159:189193.

3. Dirheimer Y, Bensimon C, Christmann D, Wackenheim C. Syndesmo-odontoid joint and calcium pyrophosphate dihydrate deposition disease (CPPD) Neuroradiology. 1983;25:319-321. 